\title{
ZEROS OF DERIVATIVES OF ENTIRE FUNCTIONS
}

\author{
THOMAS CRAVEN, GEORGE CSORDAS AND WAYNE SMITH
}

\begin{abstract}
It is shown that if a real entire function of genus one has only finitely many nonreal zeros, then, its derivatives, from a certain one onward, have only real zeros.
\end{abstract}

A real entire function $\psi(x)$ is said to be in the Laguerre-Pólya class if $\psi(x)$ can be expressed in the form

$$
\psi(x)=c x^{n} e^{-\alpha x^{2}+\beta x} \prod_{k=1}^{\infty}\left(1+x / a_{k}\right) e^{-x / a_{k}},
$$

where $c, \beta, a_{k}$ are real, $\alpha \geq 0, n$ is a nonnegative integer, and $\sum a_{k}^{-2}<\infty$ (see [L, P1]). If $\psi(x)$ is in the Laguerre-Pólya class, we will write $\psi \in \mathcal{L}-\mathcal{P}$. Of particular importance is the fact that such a function can be uniformly approximated on compact subsets of the complex plane by a sequence of polynomials with only real zeros. We shall use the notation $\mathcal{L}-\mathcal{P}^{*}$ to denote the set of all entire functions which arise as products of real polynomials and functions in $\mathcal{L}-\mathcal{P}$.

A fifty-five year old conjecture of Pólya [P2] and Wiman [W1] states that the derivatives $\varphi^{(n)}(x)$ for $\varphi(x) \in \mathcal{L}-\mathcal{P}^{*}$ will have only real zeros for all sufficiently large $n$. All work on this problem depends heavily on the order of $\varphi(x)$. (The corresponding statement fails for some functions of order 2 such as $\exp \left(x^{2}\right)$.) The first partial result was proved by Ålander in 1930 for functions of order less than $\frac{2}{3}$ [A2] and later extended by Wiman to functions of order at most 1 [W2]. In 1937, Pólya extended these results to functions of order less than $\frac{4}{3}$ [P3]. Recently, the present authors have proved the conjecture for functions of order less than 2 [CCS]. In this paper, we first obtain a refinement of an old theorem of Ålander [A1] which was needed in [A2] and [CCS]. We use this improved result to extend our proof of the Pólya-Wiman conjecture to functions of minimal type of order 2 (for definitions, see [B]).

THEOREM 1. Suppose that $f(z)=\sum b_{k} z^{k}$ is a transcendental entire function satisfying

$$
M(r)=\max _{|z|=r}|f(z)| \leq e^{c r^{d}} \quad \text { for all } r \geq r_{0},
$$

where $c$ and $d$ are positive constants. Let $\varepsilon>0$. Then there are infinitely many positive integers $n$ such that if $f^{(n)}\left(z_{n}\right)=0$, then

$$
\left|z_{n}\right|>(\log 2) e^{-1}(c+\varepsilon)^{-1 / d} n^{-1+1 / d} .
$$

Received by the editors June 30, 1986. Presented at the 832nd meeting of the AMS in Honolulu, Hawaii, March 26-28, 1987.

1980 Mathematics Subject Classification (1985 Revision). Primary 30D15, 30D35.

Key words and phrases. Pólya-Wiman conjecture, Laguerre-Polya class. 
This theorem is an improvement of Ålander's theorem (see, for example, [CCS]). Alander's theorem applied to $f(z)$ would yield, in place of $(1)$, the inequality $\left|z_{n}\right|>$ $(\log 2) n^{-1+1 / \lambda}$ for any $\lambda>d$.

PROOF OF THEOREM 1 . We have by Cauchy's inequality that

$$
\left|b_{n}\right| \leq \frac{e^{\log M(r)}}{r^{n}}, \quad n \geq 0, r>0 .
$$

Let $H(r)$ denote the inverse function of $\log M(r)$. It follows that for $r \geq c r_{0}^{d}=r_{1}$,

$$
r^{1 / d} c^{-1 / d} \leq H(r)
$$

If $r=H(n)$, then by (2), $\left|b_{n}\right| \leq(e / H(n))^{n}$. Thus, by (3), there is a positive integer $n_{1}$ such that $\left|b_{n}\right| \leq\left(e n^{-1 / d}\right)^{n} c^{n / d}$ for $n \geq n_{1}$. In particular, if $\delta>0$, then $\left|b_{n}\right| n^{n / d} e^{-n}(c+\delta)^{-n / d}=o(1)$ as $n \rightarrow \infty$. Hence there are arbitrarily large $n$ such that

$$
\left|b_{k+n} / b_{n}\right|<e^{k}(c+\delta)^{k / d}(k+n)^{-k / d}, \quad k \geq 1 .
$$

Then by (4), there are infinitely many $n$ such that

$$
\begin{aligned}
\left|\frac{f^{(n)}(z)}{n ! b_{n}}\right| & =\left|\sum_{k=0}^{\infty}\left(\begin{array}{c}
n+k \\
k
\end{array}\right) \frac{b_{k+n}}{b_{n}} z^{k}\right| \\
& \geq 1-\sum_{k=1}^{\infty}\left(\begin{array}{c}
n+k \\
k
\end{array}\right)\left|\frac{b_{k+n}}{b_{n}}\right|\left|z^{k}\right| \\
& >1-\sum_{k=1}^{\infty}\left(\begin{array}{c}
n+k \\
k
\end{array}\right) \frac{e^{k}(c+\delta)^{k / d}}{(n+k)^{k / d}}\left|z^{k}\right| \\
& >2-\sum_{k=0}^{\infty}\left(\begin{array}{c}
n+k \\
k
\end{array}\right) \frac{e^{k}(c+\delta)^{k / d}}{n^{k / d}}\left|z^{k}\right| .
\end{aligned}
$$

If $|z| e(c+\delta)^{1 / d} n^{-1 / d}<1$, then the expression above is equal to

$$
2-\left(1-e n^{-1 / d}(c+\delta)^{1 / d}|z|\right)^{-n-1} \text {. }
$$

Also, for sufficiently large $n$,

$$
(\log 2) e^{-1}(c+3 \delta)^{-1 / d} n^{-1+1 / d}<(\log 2) e^{-1}(c+2 \delta)^{-1 / d} n^{1 / d}(n+1)^{-1} .
$$

We conclude that if $|z| \leq(\log 2) e^{-1}(c+3 \delta)^{-1 / d} n^{-1+1 / d}$ and $n$ is large, then expression (5) is larger than

$$
2-\left(1-\frac{\log 2}{n+1}\left(\frac{c+\delta}{c+2 \delta}\right)^{1 / d}\right)^{-n-1} .
$$

Since (6) is positive for all sufficiently large $n$, the theorem follows by setting $\delta=$ $\varepsilon / 3$.

We now present an example showing that Theorem 1 is essentially sharp. Let $f(z)=e^{-c z^{2}}$, where $c>0$. Then $f^{(n)}$ is an odd function if $n$ is odd, so $f^{(n)}(0)=0$. Also, if $n$ is even there is a zero $z_{n}$ of $f^{(n)}$ satisfying

$$
c^{-1 / 2} n^{-1 / 2} \leq z_{n} \leq(3 / 2)^{1 / 2} c^{-1 / 2} n^{-1 / 2} \text {. }
$$


These estimates are due to Wiman [W1]. From this it is clear that the only possible improvement in inequality (1) would be an improvement in the constant $(\log 2) / e$.

An immediate consequence of Theorem 1 (with $d=2, \varepsilon$ replaced by $\varepsilon / 2$, and $c=\alpha+\varepsilon / 2)$ for functions in the Laguerre-Pólya class is the following corollary.

COROLlaRY 1. Suppose that

$$
f(z)=p(z) e^{-\alpha z^{2}+\beta z} \prod\left(1+z / a_{k}\right) e^{-z / a_{k}}
$$

is a transcendental function in $\mathcal{L}-\mathcal{P}^{*}$, where $p(z)$ is a real polynomial, and that $\varepsilon>0$. Then there are infinitely many positive integers $n$ such that if $f^{(n)}\left(z_{n}\right)=0$, then

$$
\left|z_{n}\right|>(\log 2) e^{-1}(\alpha+\varepsilon)^{-1 / 2} n^{-1 / 2} .
$$

We now turn our attention to the Pólya-Winman conjecture. We shall need the following results, in which we write $D^{n} f$ for $f^{(n)}$.

LEMMA 1 [CCS, LEMMA 1]. If $\varphi \in \mathcal{L}-\mathcal{P}^{*}$ and if $D^{m} \varphi \in \mathcal{L}-\mathcal{P}$ for some nonnegative integer $m$, then for any $a \in \mathbf{R}$

$$
D^{m+1}[(x+a) \varphi(x)] \in \mathcal{L}-\mathcal{P} \text {. }
$$

LEMMA 2 [CCS, LEMMA 2]. Let $\varphi(x)=p(x) \psi(x) \in \mathcal{L}-\mathcal{P}^{*}$, where $p(x)$ is a nonconstant polynomial with only nonreal zeros and where

$$
\psi(x)=c x^{n} e^{\beta x} \prod_{k=1}^{\infty}\left(1+x / a_{k}\right) e^{-x / a_{k}}
$$

is in $\mathcal{L}-\mathcal{P}$. Then there is a positive integer $N$ and an open nonempty interval $I$ such that if $\gamma \in I$, then $(D+\gamma) \varphi_{N}(x)$ has fewer nonreal zeros than $p(x)$, where

$$
\varphi_{N}(x)=c p(x)\left(\exp \left\{\left(\beta-\sum_{k=1}^{N-1} \frac{1}{a_{k}}\right) x\right\}\right) \prod_{k=N}^{\infty}\left(1+\frac{x}{a_{k}}\right) e^{-x / a_{k}} .
$$

THEOREM 2. Let $\varphi \in \mathcal{L}-\mathcal{P}^{*}$. If $\gamma_{1}<\gamma_{2}$ and if $\left(D+\gamma_{j}\right) \varphi(x) \in \mathcal{L}-\mathcal{P}, j=1,2$, then

$$
(D+\gamma) \varphi(x) \in \mathcal{L}-\mathcal{P} \quad \text { for all } \gamma \in\left[\gamma_{1}, \gamma_{2}\right] .
$$

Moreover, the real zeros of $D\left(\varphi^{\prime} / \varphi\right)$ are all simple.

This theorem, but with a restriction on the order of $\varphi$, is [CCS, Corollary 1]. The theorem is proved by using the same counting argument as in the original proof, except that a refinement of [CCS, Lemma 3] is now required. The upper bound in that lemma must be recognized to be $2 d+1$ when $\varphi$ has infinitely many zeros.

THEOREM 3. Let $\varphi(x)=p(x) e^{\beta x} \Pi\left(1+x / a_{k}\right) e^{-x / a_{k}} \in \mathcal{L}-\mathcal{P}^{*}$, where $p(x)$ is a polynomial. If $(D+\gamma) \varphi(x) \in \mathcal{L}-\mathcal{P}$ for all $\gamma$ in an open nonempty interval $I$, then there is a positive integer $m$ such that $D^{m} \varphi(x) \in \mathcal{L}-\mathcal{P}$.

This theorem but with a restriction to order less than 2 , was proved as Theorem 2 of [CCS]. It was proved by obtaining a contradiction involving Ålander's theorem. The same proof yields Theorem 3 when we use Corollary 1 (with $\alpha=0$ and $\varepsilon$ sufficiently small) in place of Alander's theorem. It is important to notice that Theorem 1 is translation invariant.

We now give our application of Theorem 1 to the Pólya-Wiman conjecture. 
THEOREM 4. If $p(x)$ is a polynomial and $\varphi(x)=p(x) e^{\beta x} \prod\left(1+x / a_{k}\right) e^{-x / a_{k}} \in$ $\mathcal{L}-\mathcal{P}^{*}$, then there is a positive integer $M$ such that $D^{M} \varphi(x) \in \mathcal{L}-\mathcal{P}$.

ProOF. We may assume that $p(x)$ has no real zeros and has degree $2 d$. If $d=1$, we obtain the conclusion by applying Lemma 2 , Theorem 3 , and Lemma 1 in that order. For $d>1$, we proceed by induction. Apply Lemma 2, obtaining $\gamma_{1}<\gamma_{2}$ such that $\left(D+\gamma_{j}\right) \varphi_{N}$ have less than $2 d$ nonreal zeros. By the induction hypothesis, there exists a number $r$ such that $D^{r}\left(D+\gamma_{j}\right) \varphi_{N}$ is in $\mathcal{L}-\mathcal{P}$. By Theorem $2,(D+\gamma)\left(D^{r} \varphi_{N}\right)$ is in $\mathcal{L}-\mathcal{P}$ for all $\gamma$ in $\left(\gamma_{1}, \gamma_{2}\right)$. Therefore by Theorem 3 , there exists an $m$ such that $D^{m+r} \varphi_{N}$ is in $\mathcal{L}$ - $\mathcal{P}$. An appeal to Lemma 1 completes the proof.

\section{REFERENCES}

[A1] M. Ålander, Sur le déplacement des zéros des fonctions entières par leur dérivation, dissertation, Upsala, 1914.

[A2] _ Sur les dérivées successives des fonctions régulières, Opuscula Mathematica A. Wiman Dedicata, Lund, 1930, pp. 79-98.

[B] R. P. Boas, Entire functions, Academic Press, New York, 1954.

[CCS] T. Craven, G. Csordas, and W. Smith, The zeros of derivatives of entire functions and the Pólya-Wiman conjecture, Ann. of Math. (2) 125 (1987), 405-431.

[L] E. Laguerre, Oeuvres, vol. 1, Gauthier-Villars, Paris, 1898.

[P1] G. Pólya, Über Annäherung durch polynome mit lauter reellen Wurzeln, Rend. Circ. Mat. Palermo 36 (1913), 279-295.

[P2] _ Some problems connected with Fourier's work on transcendental equations, Quart. J. Math. Oxford Ser. 1 (1930), 21-34.

[P3] — Über die Realität der Nullstellen fast aller Ableitungen gewisser ganzer Funktionen, Math. Ann. 114 (1937), 622-634.

[W1] A. Wiman, Über eine asymptotische Eigenschaft der Ableitungen der ganzen Funktionen von den Geschlechtern 1 und 2 mit einer endlichen Anzahl von Nullstellen, Math. Ann. 104 (1930), 169-181.

[W2] _ U Über die Realität der Nullstellen fast aller Ableitungen gewisser ganzer Funktionen, Math. Ann. 114 (1937), 617-621.

Department of Mathematics, University of HaWAil, Honolulu, HaWAil 96822 